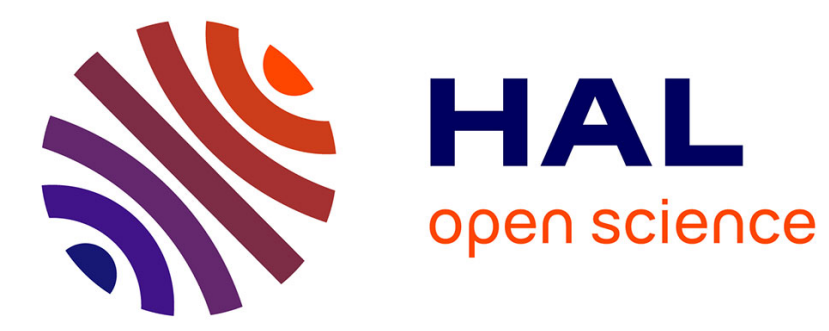

\title{
ESP performance for various dust densities
}

\author{
J. Podlinski, A. Niewulis, J. Mizeraczyk, Pierre Atten
}

\section{To cite this version:}

J. Podlinski, A. Niewulis, J. Mizeraczyk, Pierre Atten. ESP performance for various dust densities. Journal of Electrostatics, 2008, 66, pp. 246-253. hal-00372081

\section{HAL Id: hal-00372081 https://hal.science/hal-00372081}

Submitted on 31 Mar 2009

HAL is a multi-disciplinary open access archive for the deposit and dissemination of scientific research documents, whether they are published or not. The documents may come from teaching and research institutions in France or abroad, or from public or private research centers.
L'archive ouverte pluridisciplinaire HAL, est destinée au dépôt et à la diffusion de documents scientifiques de niveau recherche, publiés ou non, émanant des établissements d'enseignement et de recherche français ou étrangers, des laboratoires publics ou privés. 


\title{
ESP Performance for Various Dust Densities
}

\author{
J. Podlinski ${ }^{1}$, A. Niewulis ${ }^{1}$, J. Mizeraczyk $^{1,2}$, P. Atten ${ }^{3}$ \\ ${ }^{1}$ Centre for Plasma and Laser Engineering, The Szewalski Institute of Fluid Flow \\ Machinery, Polish Academy of Sciences, Fiszera 14, 80-952 Gdańsk, Poland \\ (janusz@imp.gda.pl) \\ ${ }^{2}$ Department of Marine Electronics, Gdynia Maritime University, Morska 81-87, \\ 81-225 Gdynia, Poland \\ ${ }^{3}$ LEMD, CNRS and Joseph Fourier University, BP 166, 38042 Grenoble Cedex 9, France
}

\begin{abstract}
In this paper, results of Particle Image Velocimetry (PIV) measurements of the electrohydrodynamic (EHD) flow velocity fields in an electrostatic precipitator (ESP) are presented. The measurements were carried out for various densities of submicron dust. The ESP was an acrylic parallelepiped with a wire discharge electrode and two plate collecting electrodes. The positive DC voltage was applied to the wire electrode, placed perpendicularly to the main gas flow. The PIV measurements were carried out in a plane placed perpendicularly to the wire electrode at its half-length. The obtained results show that the general shape of the flow patterns at different dust densities is similar in the bulk of the flow. However, the flow velocity and the level of its turbulence strongly depend on the dust density, in particular in the region around the wire electrode.
\end{abstract}

\section{Introduction}

For several decades electrostatic precipitators (ESPs) have been widely used as dust particle collectors. They are characterized by a high total particle collection efficiency (up to $99.9 \%$ ) with a low pressure drop. However, there is still a problem with collection efficiency 
of submicron particles [1], which can contain traces of toxic elements. Moreover, submicron particles float relatively long in the atmosphere and can easily penetrate into human respiratory system. Therefore, there has long been an interest in improving the ESP collection of submicron particles.

The precipitation of particles in the duct of an ESP depends on the dust-particle properties, electric field, space charge and flow pattern [2]. The influence of the electrohydrodynamic (EHD) secondary flow, formed electrically in ESPs, on the collection efficiency has been debated for decades. The flow patterns in ESPs were studied using various visualization and laser techniques [e.g., 3-7]. Recently, the Particle Image Velocimetry (PIV) [8,9] was used in investigations of the flow fields in ESPs [10-13]. To elucidate the influence of the electrically generated flow disturbances on the gas cleaning process, also numerical modelling investigations were carried out [e.g., 14-16]. The results of the numerical modelling of particle collection efficiency showed that the particle collection could be significantly improved if the EHD secondary flow were eliminated [14]. However, study of the influence of the EHD secondary flow on the collection efficiency is difficult because the EHD flow is complex due to its stochastic nature. Besides the EHD secondary flow depends not only on the primary flow and electric field, but also on the dust-particle properties, including the dust density.

In this paper, the results of PIV measurements of the flow velocity fields in a wire-plate ESP for various dust densities are presented.

\section{Experimental apparatus}

The apparatus used in this experiment consisted of an ESP, a high voltage supply and a standard PIV equipment for the measurement of velocity fields (Fig. 1). The ESP was an acrylic parallelepiped $1000 \mathrm{~mm}$ long, $200 \mathrm{~mm}$ wide and $100 \mathrm{~mm}$ high. The electrical electrode set consisted of two collecting plate electrodes and a discharge wire electrode. The 
wire electrode (diameter $1 \mathrm{~mm}$, length $200 \mathrm{~mm}$ ) and the plate electrodes $(200 \mathrm{~mm}$ wide and $600 \mathrm{~mm}$ long) were made of stainless steel. The wire electrode was placed midway between the collecting plate electrodes, perpendicularly to the main flow direction (Fig. 1). The distance between the wire and the collecting electrodes was $50 \mathrm{~mm}$. A flow homogenizer was placed before the ESP inlet. The ESP together with the flow homogenizer was a part of an aerodynamic loop with recirculation of a finite volume $\left(\sim 2 \mathrm{~m}^{3}\right)$ of air.

A positive DC voltage of $30 \mathrm{kV}$ was applied to the wire electrode through a $10 \mathrm{M} \Omega$ resistor, while the plate electrodes were grounded. The averaged discharge current strongly depended on the dust density and varied from $90 \mu \mathrm{A}$ (for very high dust density) to $160 \mu \mathrm{A}$ (for very low dust density).

The dry air in the aerodynamic loop was blown so that the air average velocity in the ESP was $0.4 \mathrm{~m} / \mathrm{s}$. Cigarette smoke was introduced into the aerodynamic loop to get dust particles in the ESP. The characterization of the smoke was performed in another aerodynamic loop set-up based on a balloon vessel of about $2.5 \mathrm{~m}$ in diameter $\left(\sim 8 \mathrm{~m}^{3}\right)$. The balloon was first filled with filtered air, which was pushed by a fan through a box where cigarettes were burnt. Then the formed air-cigarette smoke mixture flowed in the aerodynamic loop. The measurement of the cigarette particle concentrations in the air-smoke flow was carried out by classes of size using an optical counter (TOPAS LAP320) on scales ranging from $0.31 \mu \mathrm{m}$ to $20 \mu \mathrm{m}$. The particle size distributions are shown in Fig. 2 for several numbers of simultaneously burnt cigarettes. Fig. 2 shows a suspension of very fine particles existed in the flow. Most particles had a diameter lower than $1 \mu \mathrm{m}$. As expected, the particle concentration increased with the number of simultaneously burnt cigarettes. For 8 and 14 burnt cigarettes, the particle size distribution curves exhibit a maximum at a particle diameter $d_{p} \cong 0.4 \mu \mathrm{m}$. The mean size tends to increase with increasing total particle concentration, presumably due to an 
agglomeration of fine particles. We estimate that the mass concentration of the particles ranged from a few $\mathrm{mg} / \mathrm{m}^{3}$ to a few tens of $\mathrm{mg} / \mathrm{m}^{3}$.

The PIV measurementswere carried out for various particle densities: very low, low, medium, high, and very high (the density was controlled by a number of simultaneously burnt cigarettes). The ratio between the particle densities was estimated by comparing the averaged brightness of the flow images (without applied voltage) taken for each dust density. The dust density ratio was approximately 1:2:4:8:16 for the very low:low:medium:high:very density of the dust. In this paper only results for the very low, medium, and very high dust densities are presented.

The standard PIV equipment consisted of a twin second harmonic Nd-YAG laser system $(\lambda=532 \mathrm{~nm}$ ), imaging optics (cylindrical telescope), CCD camera and PC computer (Fig. 1). The laser sheet of thickness of $1 \mathrm{~mm}$, formed from the Nd-YAG laser beam by the cylindrical telescope was introduced into the ESP to form an observation plane. The images of the particles in the laser sheet were recorded by FlowSense M2 camera. The camera was equipped with interference filter (FWHM $11.8 \mathrm{~nm}$ around $\lambda=532.6 \mathrm{~nm}$ ) to eliminate the influence of ambient light on the image brightness. The CCD camera active element size was $1186 \times 1600$ pixels. The captured images were transferred to the PC computer for digital analysis.

The PIV measurements were carried out in a plane placed perpendicularly to the wire electrode at its half-length (Fig. 1). The observation area (the area of the laser sheet "seen" by the CCD camera) covered a region between the plate electrodes, ranging from $15 \mathrm{~mm}$ in the flow upstream direction to $205 \mathrm{~mm}$ in the flow downstream direction, when measured from the wire electrode (Fig. 3). The image of the observation area were taken by the CCD camera with a resolution $730 \times 1600$ pixels. 
Each velocity field presented in this paper resulted from averaging of 150 PIV images, which means that each velocity map was time-averaged. Based on the measured velocity fields, the corresponding mean flow streamlines in the observation area were determined.

\section{Results}

Figs. 4-7 show the results of the PIV measurements in the ESP when no voltage was applied. The Reynolds number was $R e=V \times L / v=2550$ [17]. The parameters used to calculate $R e$ were: the primary flow average velocity $V=0.4 \mathrm{~m} / \mathrm{s}$, the characteristic length (plate-plate distance) $L=0.1 \mathrm{~m}$, and the air kinematic viscosity $v=1.57 \times 10^{-5} \mathrm{~m}^{2} / \mathrm{s}$ ).

Fig. 4 shows a typical image of the flow in the ESP taken by the CCD camera for the medium dust density. The dust particles scattering the laser light are visible as bright points in the image. Although a difference in the image brightness can be seen between the centre of the ESP channel and the regions near the plate electrodes, we assess that the dust distribution was uniform in the ESP. The brightness difference resulted from the non-uniform transverse intensity distribution of the laser sheet produced from the laser beam which had Gaussian profile of light intensity. There is also some difference in the brightness between the left and right sides of the image. The decrease in the laser sheet intensity along its propagation direction (from left to right in the image) was partly due to loss of the laser beam intensity caused by the scattering of the laser light by the dust particles. The brightness variation was also due to the laser light scattering profile on the dust particles: the camera "sees" the forward scattered light at the image left side, the perpendicular scattered light in the centre, and the back scattered light at the image right side.

When no voltage was applied, the submicron dust particles followed the gas main flow and, in this case, the measured velocity field of the particles corresponds to the gas flow velocity field. Figs. 5-7 show the particle mean velocity field, the corresponding flow streamlines and the particle flow velocity standard deviation in the ESP for the medium dust 
density, when no voltage was applied. It is clearly seen from these figures that the flow was laminar and the flow velocity standard deviation was very low $(+/-0.03 \mathrm{~m} / \mathrm{s})$. A small decrease in the flow velocity was found in the wake behind the wire electrode. The flow velocity patterns for the very low and the very high dust densities (not shown in this paper) when no voltage was applied, were very similar to that for the medium dust density.

When a high voltage was applied to the wire electrode, the corona discharge on the wire was creating a cloud of positive ions. In clean gases the mechanism of corona discharge involves different processes occurring in a thin layer (high field zone around the wire). These physical processes are well known [18] and involve various phenomena like molecules ionization by accelerated electrons, electrons avalanches, electron-ion recombination and photons emission, electron attachment by electronegative species, secondary electron emission by a cathode through bombardment by positive ions, photo-electric effect on the gaseous molecules, etc.. These processes are extremely difficult to model. In practical situations, the modelling is highly simplified by neglecting the thickness of the ionisation layer and considering only one ionic species $[19,20]$. The crucial point then is to formulate the boundary condition for the space charge density. The so-called Kaptzov assumption $[21,22]$ is commonly accepted which states that the field value on the wire is constant whatever the current (this arises because the ionization dramatically depends on the electric field value see $[18,22])$. Most often the retained field value is the one $E_{P e e k}$ proposed by Peek [23].

For a given space charge distribution, the field on the wire $E_{\text {wire }}$ is the sum of the harmonic field and of the field component $\delta E_{\rho}$ due to the space charge (which diminishes the field amplitude) :

$$
E_{\text {wire }}=E_{\text {harm }}+\delta E_{\rho}
$$

The space charge induced field modification $\delta E_{\rho}$ depends linearly on the charge density : $\delta E_{\rho}$ $\propto\left(\bar{\rho}_{\text {ion }}\right)_{0}$ where $\left(\bar{\rho}_{\text {ion }}\right)_{0}$ is the mean ionic space charge density in clean air (obtained by 
averaging on an adequate domain of finite extent). Note that in the numerical modelling for ionizing wires $[19,20]$, the injected space charge density $\rho_{i n j}$ on the wire is modified until the resulting field $E_{\text {wire }}$ equals $E_{\text {Peek }}$.

In dusty gases, the ions drifting between the wire and the two plate electrodes are charging the dust particles. Therefore, in our experiments, the space charge had two components, one, $\rho_{\text {ion }}$, due to the ions and the other one, $\rho_{p}$, corresponding to the charged dust particles. Now, the physical processes occurring in the corona sheath around the wire are not affected by the presence of dust particles (Fig. 8 shows that the particles don't enter this sheath). Therefore the field conditions controlling the corona discharge on the wire remain unchanged and, for a given applied voltage, $\delta E_{\rho}=$ Cst, independently of the dust concentration $c$. Although the space charges of ions $\rho_{\text {ion }}$ and of particles $\rho_{p}$ have not a priori the same spatial distributions, we assume that, to a first approximation, their influences are similar; then the condition $\delta E_{\rho}=$ Cst leads to :

$$
\bar{\rho}_{\text {total }}=\bar{\rho}_{i o n}+\bar{\rho}_{p} \cong C s t=\left(\bar{\rho}_{\text {ion }}\right)_{0}
$$

This relation implies that an increase in the mean charge density $\bar{\rho}_{p}$ of charged particles should be compensated for by a decrease in the mean ionic charge density $\bar{\rho}_{\text {ion }}$.

We can get some information on the relative magnitude of particles and ions mean charge densities from the change in total current $I$; outside the corona sheath where there are electrons, the current density $\boldsymbol{j}$ is the sum of the contributions due to ions $\left(\boldsymbol{j}_{\text {ion }}\right)$ and charged particles $\left(\boldsymbol{j}_{p}\right)$. As the mobility $K_{\text {ion }}$ of ions is much higher than the one, $K_{p}$, of submicron particles, $j_{p}=K_{p} \rho_{p} E$ is negligible compared with $j_{\text {ion }}=K_{\text {ion }} \rho_{\text {ion }} E$. In practice, the measured total current, equal to the integral of $j$ on the collecting plates, is determined by the drift of ions only :

$$
I=I_{i o n}+I_{p} \approx I_{i o n} \propto \bar{\rho}_{i o n}
$$


Therefore we can compare the global charge density $\bar{\rho}_{p}$ of charged particles and the one of ions, $\bar{\rho}_{\text {ion }}$, from the changes in the current value $I$ :

$$
\frac{\bar{\rho}_{p}}{\bar{\rho}_{i o n}} \cong \frac{\left(\bar{\rho}_{i o n}\right)_{0}-\bar{\rho}_{i o n}}{\bar{\rho}_{i o n}} \propto \frac{I_{0}-I}{I}
$$

where $I_{0}$ is the corona discharge current in air without particles. The averaged discharge current $I$ strongly depended on the dust density and it was equal to $160 \mu$ A for the very low dust density, $120 \mu \mathrm{A}$ for the medium one and $90 \mu \mathrm{A}$ for the very high dust density (see Figs. 8-12). This clearly shows that the mean ion density was decreasing with the dust density $c$ and therefore, that the density $\bar{\rho}_{p}$ of charged particles increased with $c$; in particular, for very high dust density, the mean charge densities $\bar{\rho}_{\text {ion }}$ and $\bar{\rho}_{p}$ appear to take similar values $\left(I / I_{0}\right.$ $\cong 1 / 2)$.

The electric forces exerted by the field on the space charge can induce a strong secondary flow of the gas which can alter significantly the primary laminar flow. On another hand, the electric force on each individual particle with charge $q_{p}$ induces a drift of the particle with respect to the suspending air. Therefore, the net motion of the charged dust particles has two components, one due to the gas flow and the other one, the particle drift velocity, due to the electric force $q_{p} \boldsymbol{E}$. It must be noted that the drift velocity of charged submicron particles is very limited (a few $\mathrm{cm} / \mathrm{s}$ - see [2]) so that they are easily entrained by the air flow which modifies the distribution of charged dust particles and, subsequently, the distribution of force density $\left(\rho_{\text {ion }}+\rho_{p}\right) \boldsymbol{E}$ and, hence, the gas flow itself [2].

Figs. 8-12 show the results of the dust particle flow velocity field measurements in the ESP for three different dust densities (very low, medium and very high), when a high voltage of $+30 \mathrm{kV}$ was applied. The average air flow velocity was $0.4 \mathrm{~m} / \mathrm{s}$. The particle flow velocity fields measured by PIV method (Figs. 10-12) reflect motion of the dust particles in the ESP. 
The instantaneous images of the particle flow patterns for the three dust densities shown in Figs. 8a-c were processed by the computer. The computer processing consisted in brightening up the original images so that the average brightness of all three pictures was similar regardless of the dust density. Due to this treatment, the flow patterns for the lower dust densities are more clearly visible.

As can be seen from Fig. 8a (very low dust density), after applying the voltage a dark trail with bright borders appeared behind the wire electrode. The dark trail is an area in which the dust particles were removed due to the electric force $q_{p} E$ (in this area there are no dust particles, and hence, no scattered laser light). The bright trail borders show areas with a relatively high dust density. The trail structure clearly visible behind the wire, is then scattered by a turbulent flow, about $50 \mathrm{~mm}$ behind the wire electrode $(\mathrm{x}=50 \mathrm{~mm})$ in the downstream direction.

At constant primary flow velocity and applied voltage, the dust density increase caused a decrease in the average discharge current and a change in the flow structure behind the wire electrode (Figs. $8 \mathrm{~b}$ and c). As seen, the trail type of flow structure significantly spreads out towards the collecting plate electrodes at higher dust densities. At the very high dust density (Fig. 8c), the dark trail with bright borders covered almost the whole height of the ESP channel. This means, that almost all the dust particles which flowed in the vicinity of the wire electrode were directed towards the collecting electrodes (the abundance of the dust particles is represented by the bright area from the wire electrode to the plate electrodes). About $50 \mathrm{~mm}$ behind the wire $(x=50 \mathrm{~mm})$ vortices were formed. These vortices dispersed and then scattered in the main flow. The considerable changes in the flow patterns, shown in Figs. 8 a-c, arise from the changes of the particles charge density $\rho_{p}$ and in the corresponding electric force density. Figs. 8 a-c illustrate the strong relation between the particles charge density and the gas flow structure. 
It is also seen from Figs. 8 a-c that the density of the dust inflowing into the discharge region (see the cross-sections at $\mathrm{x}=-10 \mathrm{~mm}$ ) was not uniform regardless of the dust density. The dust density was highest in the centre of the ESP duct and lowest near the plate electrodes. This was caused by large, regular vortices occurring near the plate electrodes in front of the wire electrode (see Figs. 10 and 11).

The results of the averaging of 150 instantaneous flow images in the ESP for different dust densities are shown in Figs. 9 a-c. For all the dust densities, the brightness of the presented averaged images was preserved as resulting from the original instantaneous images (in contrary to the images with corrected brightness presented in Figs. 8 a-c). Therefore, the brightness of the averaged images allows us to estimate and compare the local dust densities for the three dust density cases: very low, medium and very high. The averaged images clearly show that the particle-free region (dark area) spreads out faster with increasing dust density.

The averaged particle flow velocity fields and the corresponding flow streamlines in the observation area in the ESP are shown in Figs. 10 and 11, respectively. The particle flow velocity fields and the corresponding streamlines in Figs. 10 and 11 differ slightly from the gas flow velocity fields (and the corresponding streamlines). To obtain the gas flow patterns one has to subtract the particle drift velocity from the measured particle velocity. However, this requires a complex computing of the drift of the charged particles in the electric field with a space charge in the flowing gas. However, the drift velocity of fine particles is considerably smaller than the gas velocity in the whole ESP duct, except in the area around the wire where the electric field is very high. This means that the gas flow patterns in the ESP can be reasonably well represented by the particle flow patterns presented in Figs. 10 and 11.

As can be seen from Figs. 10 and 11, after applying the high voltage, the particle flow velocity field in the ESP changed significantly with respect to that observed without voltage. 
Vortices were formed in front of the wire electrode. They increased significantly the dust flow velocities in the central part of the ESP duct and forced a part of the dust particles to flow near the plate electrodes in the opposite direction to the main flow direction. The strong electric forces near the wire electrode moved the dust particles from the central part of the ESP channel to the plate electrodes. At the very low dust density, the dust particle movement towards the plate electrodes was smaller than for higher dust densities, although in both cases the operating voltage was the same. The differences in the particle velocities in the downstream part of ESP (e.g., $\mathrm{x}=100 \mathrm{~mm}$ ) were small. However, it is seen that the dust particle velocity slightly decreased in the ESP duct centre and increased near the plate electrodes. When the dust density is higher, the differences between the particle velocities in the downstream part of ESP increased. For very high dust density (Figs. 10c and 11c) the particle flow velocity behind the wire electrode (at $\mathrm{x}=25 \mathrm{~mm}$ ) decreased to zero. This means that almost all dust particles entering the ESP moved near the plate electrodes (with velocities up to $1 \mathrm{~m} / \mathrm{s}$ ). In the downstream ESP region the dust particle velocities became almost equal. $200 \mathrm{~mm}$ behind the wire electrode the dust particle velocities were $0.25 \mathrm{~m} / \mathrm{s}$ in the centre of the ESP duct, and $0.6 \mathrm{~m} / \mathrm{s}$ near the plate electrodes.

Fig. 12 shows the particle flow velocity standard deviation in the ESP for different dust densities. The standard deviation (in percentage) was calculated for every velocity vector as follows: Velocity std. deviation [\%] = Velocity std. deviation $/ \mid$ Velocity $\mid \cdot 100 \%$. In the ESP downstream region, near the plate electrodes the particle flow velocity standard deviation was similar for all measured dust densities; but in the centre of the ESP duct the velocity standard deviation increased with increasing dust density. This means, that at higher dust densities the flow is more turbulent in the centre of the ESP duct. 


\section{Conclusions}

The aim of the PIV measurements was to elucidate the influence of dust density on the particles flow pattern in the ESP. The results show that increasing the dust density decreases the average discharge current but increases the density of charged particles. This results in significant changes in the distribution of electric forces and, as a consequence, in the gas flow patterns.

The strong electric field around the wire induces a strong electric force on the charged dust particles which gain a drift velocity higher than the gas velocity within a distance of a few millimetres from the wire electrode, and therefore, are repelled from the wire, leaving a zone free of dust particles (the dark trail seen in Figs. 8 and 9 near the wire electrode). The analysis of the flow velocity fields shows that the increase in the dust density causes not only a significant change in the mean flow pattern (EHD secondary flow) but also results in an increase in the flow turbulence in the downstream ESP region.

Our results obtained for the very low dust density well agree with the results of EHD flow study done by T. Yamamoto and H. R. Velkoff [3], who performed Schlieren visualisation, mist particles visualisation and numerical simulation of EHD flow in a positively polarised wire-plate ESP. This agreement presumably resulted from the fact that these authors dealt also with a dust of low concentration, where the effect of particles space charge is nearly negligible. However, at higher dust densities, the PIV technique gives precise quantitative results which clearly show the importance of the particles space charge for the ESP performance.

The results suggest that, in large industrial ESPs with several collecting sections, the flow structures change in the consecutive sections due to the decrease in dust concentration. In the first section, where the dust density is relatively highest, the flow disturbances should be the strongest and a high turbulence should be generated. In the next sections the flow disturbances are expected to become less and less vigorous. 


\section{REFERENCES}

[1] A. Mizuno, Electrostatic Precipitation, IEEE Trans. Diel. Electr. Insul. 7 (2000) 615-624.

[2] P. Atten, F.M.J. McCluskey, A.C. Lahjomri, The Electrohydrodynamic Origin of Turbulence in Electrostatic Precipitators, IEEE Trans. Ind. Appl. 23 (1987) 705-711.

[3] T. Yamamoto, H. R. Velkoff, Electrohydrodynamics in an Electrostatic Precipitator, J. Fluid Mech. 108 (1981) 1-18.

[4] G. L. Leonard, M. Mitchner, S. A. Self, An Experimental Study of the Electrohydrodynamic Flow in Electrostatic Precipitators, J. Fluid Mech. 127 (1983) 123140.

[5] G.A. Kallio, D.E. Stock, Flow Visualization Inside a Wire-Plate Electrostatic Precipitator, IEEE Trans. Ind. Appl. 26 (1990) 503-514.

[6] J.H. Davidson, P.J. McKinney, EHD Flow Visualization in the Wire-Plate and Barbed Plate Electrostatic Precipitator, IEEE Trans. Ind. Appl. 27 (1991) 154-160.

[7] S.J. Park, S.S. Kim, Electrohydrodynamic Flow and Particle Transport Mechanism in Electrostatic Precipitators with Cavity Walls, Aerosol Sci. Technol. 33 (2000) 205-221.

[8] J. Westerweel, Fundamentals of Digital PIV, Meas. Sci. Technol. 8 (1997) 1379-1392.

[9] M. Raffel, Ch.E. Willert, J. Kompenhans, "Particle Image Velocimetry, A Practical Guide”, Springer-Verlag Berlin Heidelberg, 1998.

[10] J. Mizeraczyk, M. Kocik, J. Dekowski, M. Dors, J. Podliński, T. Ohkubo, S. Kanazawa, T. Kawasaki, Measurements of the Velocity Field of the Flue Gas Flow in an Electrostatic Precipitator Model Using PIV Method, J. Electrost. $51-52$ (2001) 272-277.

[11] J. Mizeraczyk, J. Dekowski, J. Podliński, M. Kocik, T. Ohkubo, S. Kanazawa, Laser Flow Visualization and Velocity Fields by Particle Image Velocimetry in Electrostatic Precipitator Model, J. Visualization 6, 2 (2003) 125-133.

[12] J. Podliński, J. Dekowski, J. Mizeraczyk, D. Brocilo, J.S. Chang, Electrohydrodynamic Gas Flow in a Positive Polarity Wire-Plate Electrostatic Precipitator and the Related Dust Particle Collection Efficiency, J. Electrost. 64 (2006) 259-262. 
[13] J. Podliński, J. Dekowski, J. Mizeraczyk, D. Brocilo, K. Urashima, J.S. Chang, EHD Flow in a Wide Electrode Spacing Spike-Plate Electrostatic Precipitator under Positive Polarity, J. Electrost. 64 (2006) 498-505.

[14] U. Kogelschatz, W. Egli, E.A. Gerteisen, Advanced Computational Tools for Electrostatic Precipitators, ABB Review 4 (1999) 33-42.

[15] D. Brocilo, J.S. Chang, R. Godard, A. Berezin, Numerical Modelling of Wire-Pipe Electrostatic Precipitator for Control of Fine Particulates, J. Aerosol Sci. 30-1 (1999) 855-856.

[16] H. Fujishima, Y. Morita, M. Okubo, T. Yamamoto, Numerical Simulation of Threedimensional Electrohydrodynamics of Spiked-Electrode Electrostatic Precipitators, IEEE Trans. Diel. Electr. Insul. 13-1 (2006) 160-167.

[17] IEEE-DEIS-EHD Technical Committee, Recommended International Standard for Dimensionless Parameters Used in Electrohydrodynamics, IEEE Trans. Diel. Electr. Insul. 10-1 (2003) 3-6.

[18] Yu.P. Raizer, “Gas discharge Physics”, Springer Verlag, Berlin, Germany, 1991.

[19] J.Q. Feng, Application of Galerkin Finite-element Method with Newton iterations in computing steady-state solutions of unipolar charge currents in corona devices, J. Comp. Phys., 151, pp. 969-989, 1999.

[20] A. M. Meroth, T. Gerber, C. D. Munz, P. L. Levin and A. J. Schwab, Numerical solution of nonstationary charge coupled problems, J. Electrostat., 45, pp.177-198, 1999.

[21] N.A. Kaptzov, Elektricheskiye Yavleniya v Gazakh i Vacuume, OGIZ, Moscow, 1947, pp. 587-630 (in Russian).

[22] K. Adamiak, V. Atrazhev and P. Atten, Corona Discharge in the Hyperbolic Point Plane Configuration : Direct Ionisation Criterion versus Approximate Formulations, IEEE Trans. Dielectrics and Electrical Insulation, 12, pp. 1015-1024, 2005.

[23] F. W. Peek, Dielectric phenomena in High Voltage Engineering, McGraw-Hill, New York, 1929. 


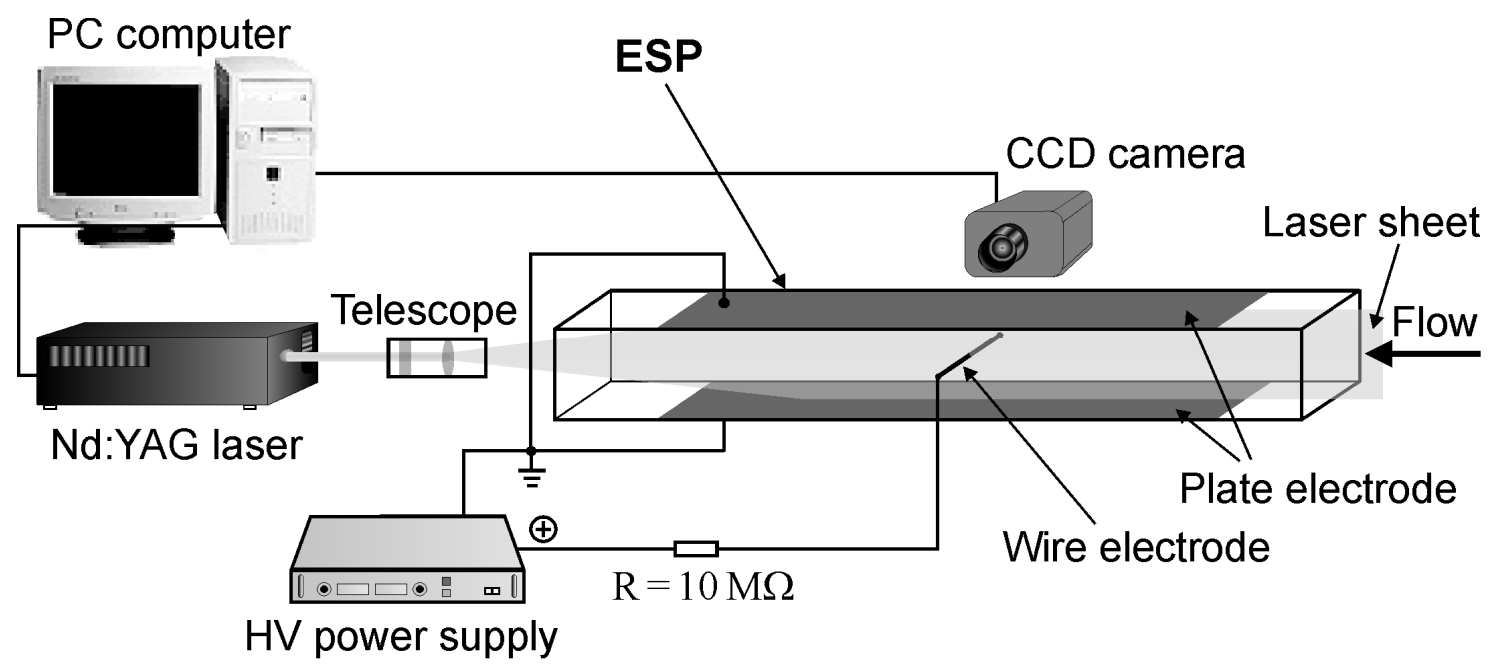

Fig. 1 - Experimental set-up for PIV flow velocity field measurements in an ESP.

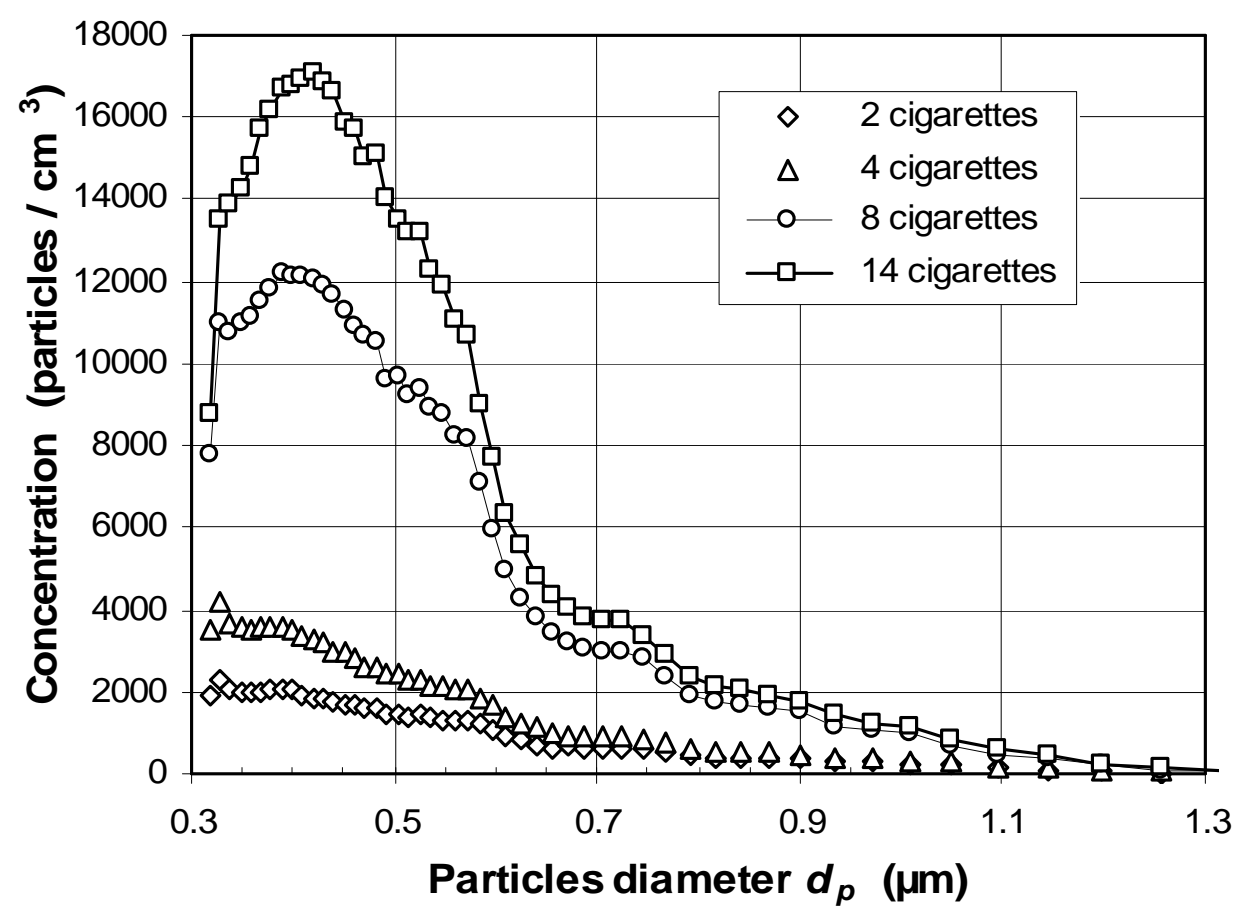

Fig. 2 - Size distributions (in particles $/ \mathrm{cm}^{3}$ ) of cigarette smoke particles for different numbers of simultaneously burnt cigarettes. 


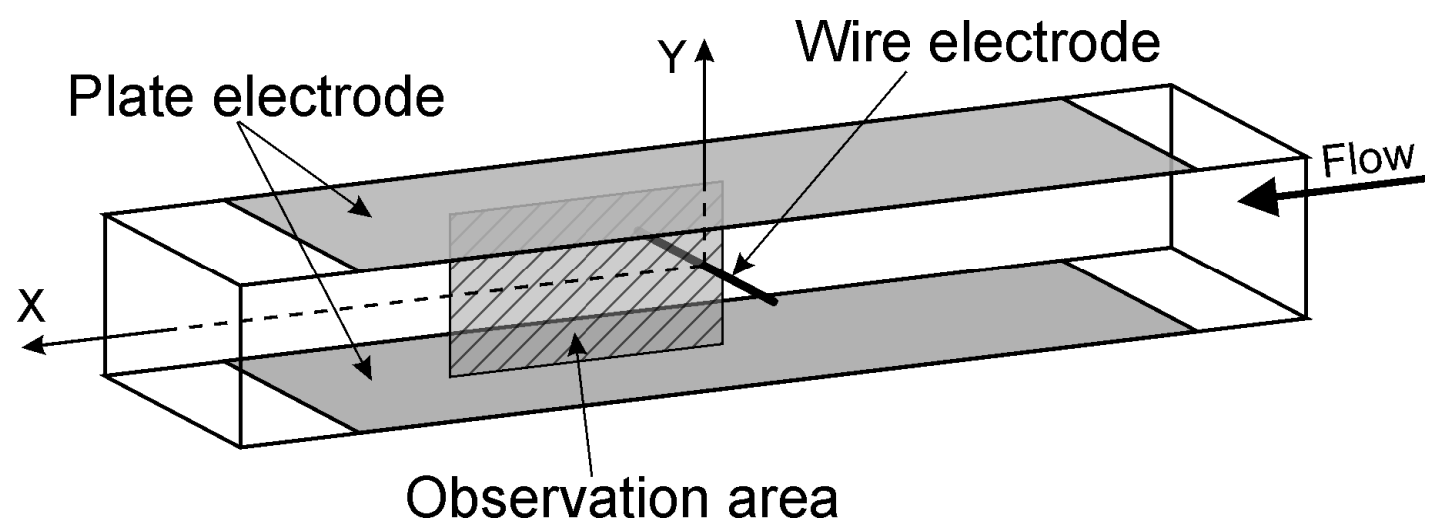

Fig. 3 - The observation area in the ESP.

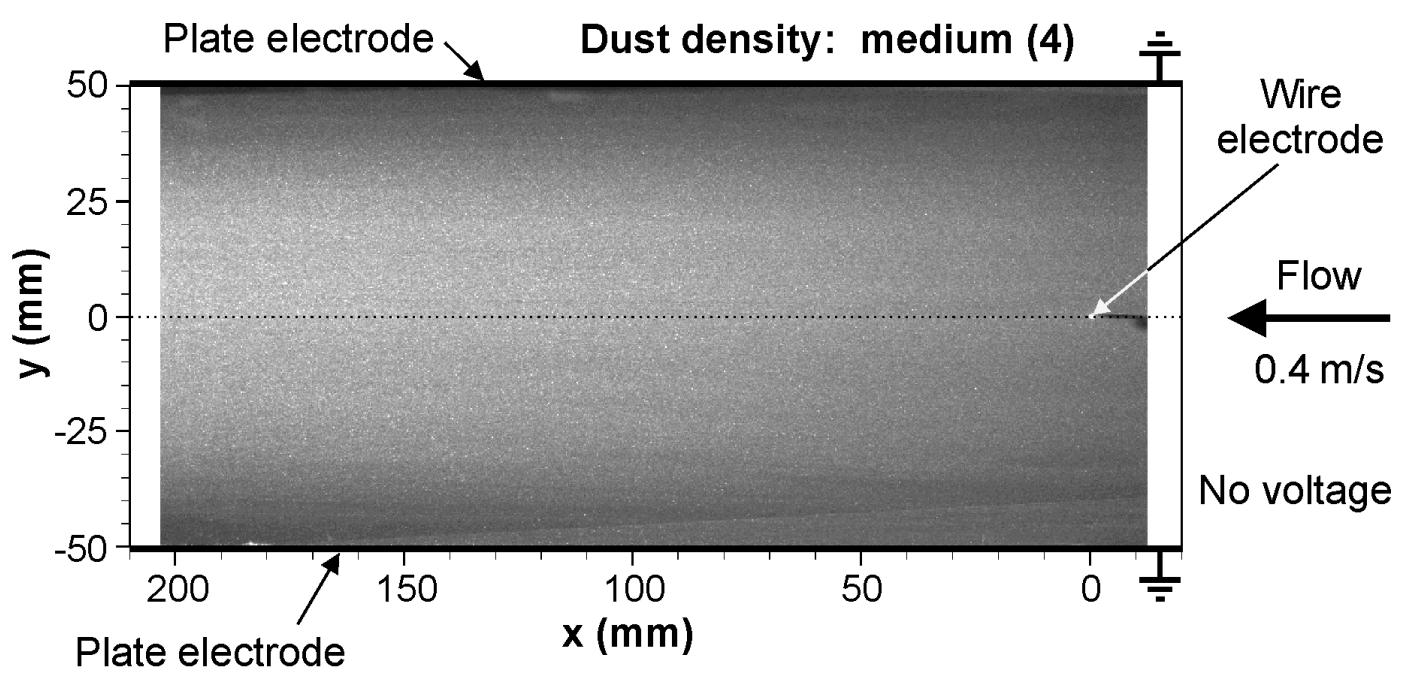

Fig. 4 - The image of the flow at the medium dust density in the ESP. The primary flow velocity was $0.4 \mathrm{~m} / \mathrm{s}$. No voltage was applied. 


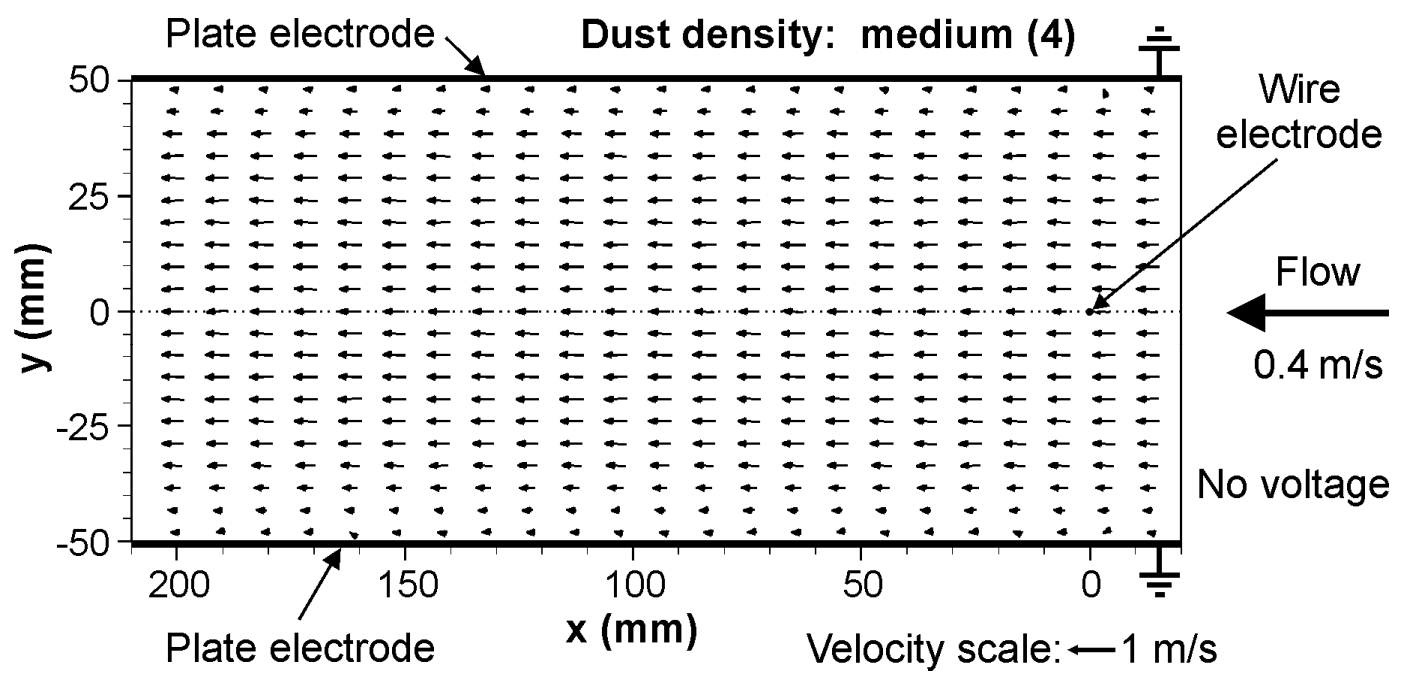

Fig. 5 - The average flow velocity field at the medium dust density in the ESP. The primary flow velocity was $0.4 \mathrm{~m} / \mathrm{s}$. No voltage was applied.

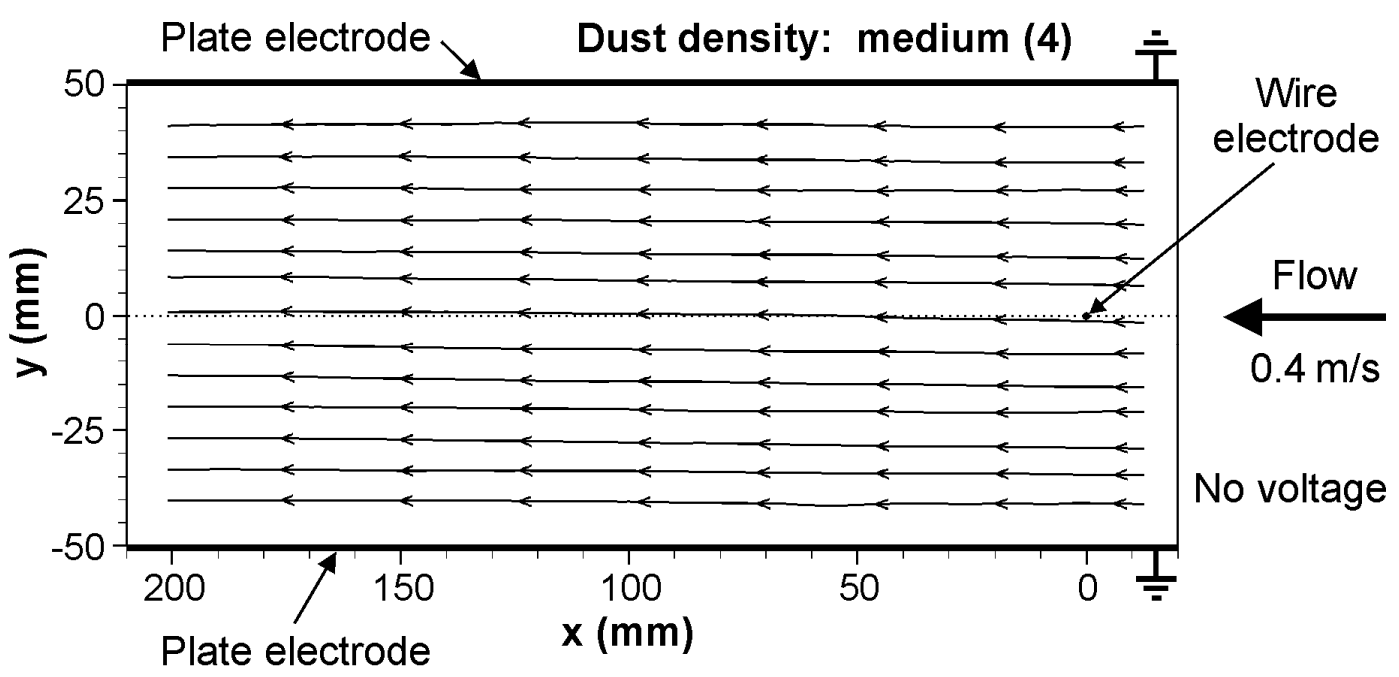

Fig. 6 - The flow streamlines at the medium dust density in the ESP. The primary flow velocity was $0.4 \mathrm{~m} / \mathrm{s}$. No voltage was applied. 


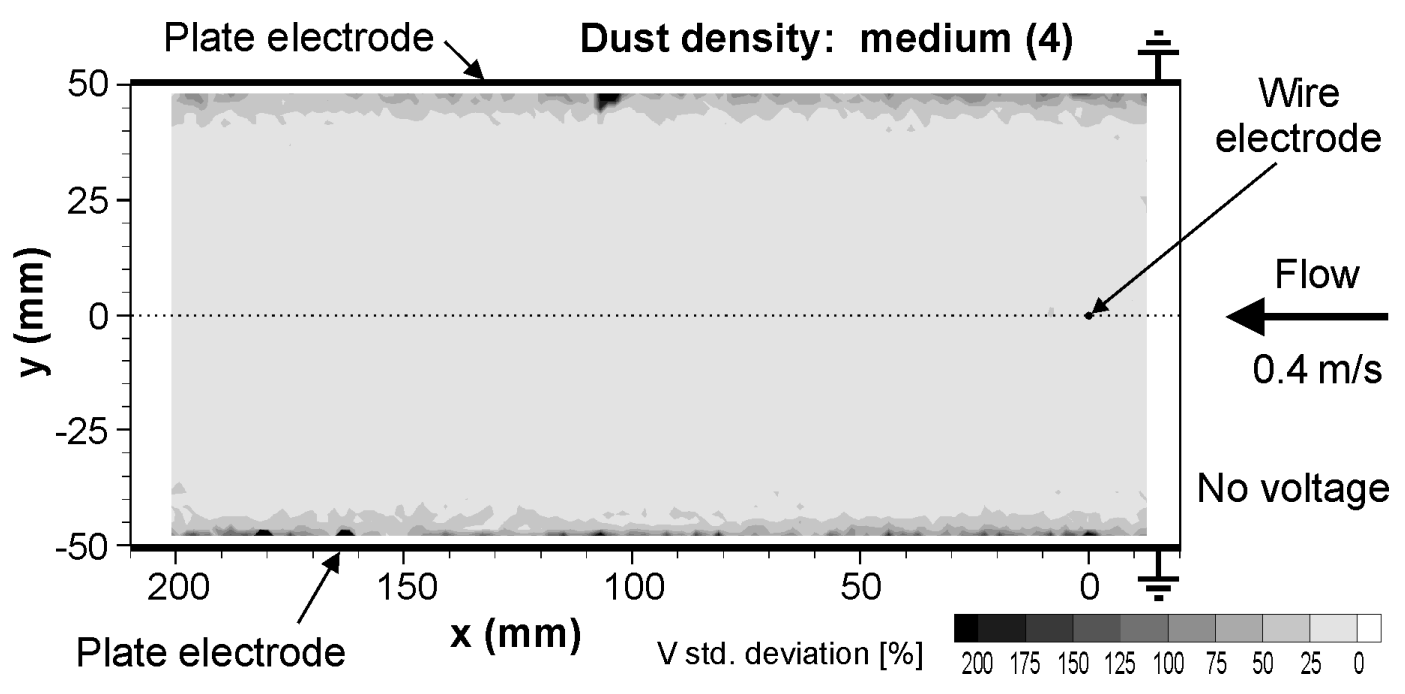

Fig. 7 - The flow velocity standard deviation at the medium dust density in the ESP. The primary flow velocity was $0.4 \mathrm{~m} / \mathrm{s}$. No voltage was applied. 

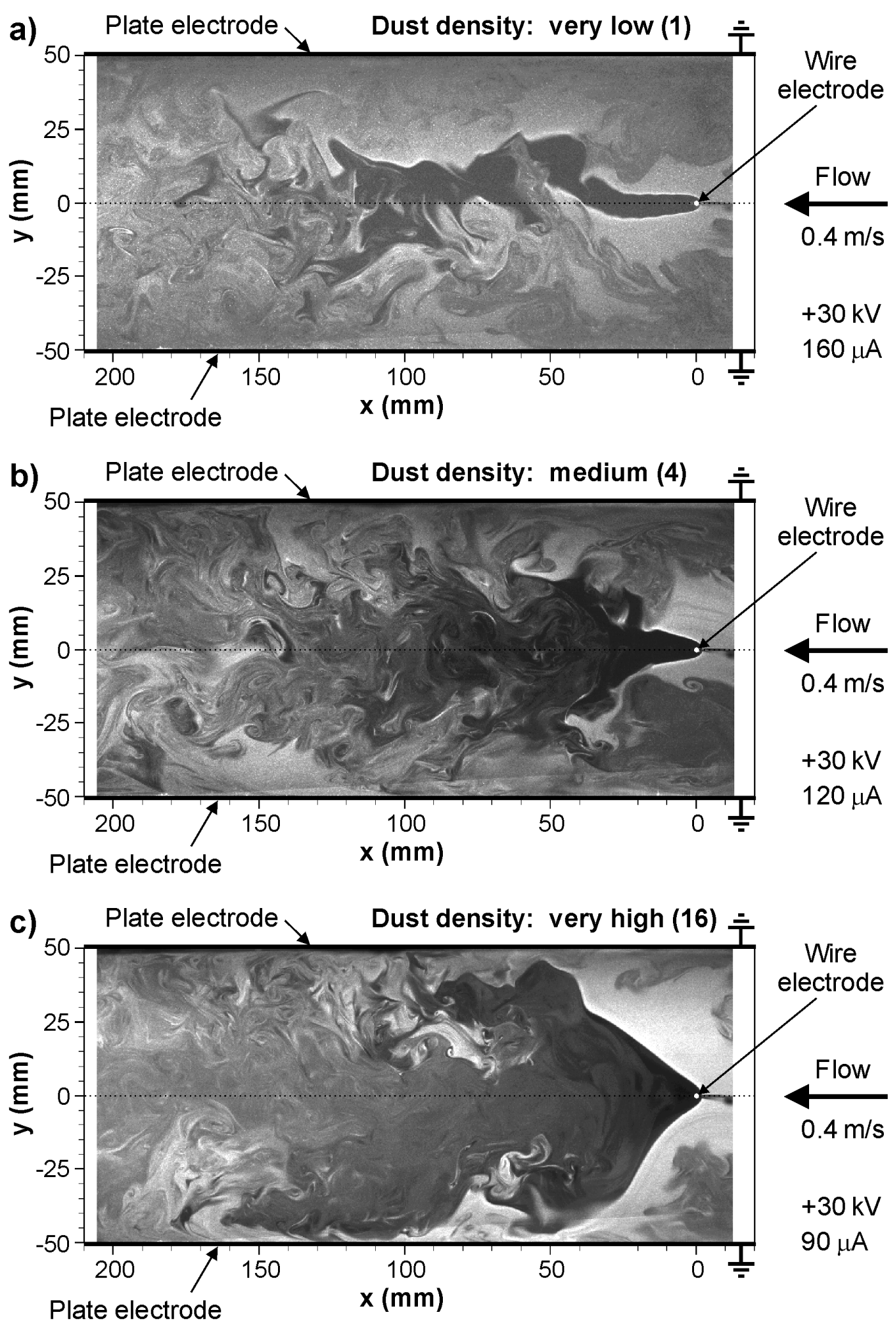

Fig. 8 - Instantaneous images of the particle flow in the ESP (primary flow velocity :

$0.4 \mathrm{~m} / \mathrm{s}$, applied voltage $:+30 \mathrm{kV}$ ). a) very low dust density (greyscale : 20), total discharge current $\mathrm{I}=160 \mu \mathrm{A} ; \quad$ b) medium dust density (greyscale : 70), $\mathrm{I}=120 \mu \mathrm{A}$; c) very high dust density (greyscale : 140), I $=90 \mu \mathrm{A}$. The bright places correspond to high density of the dust. 

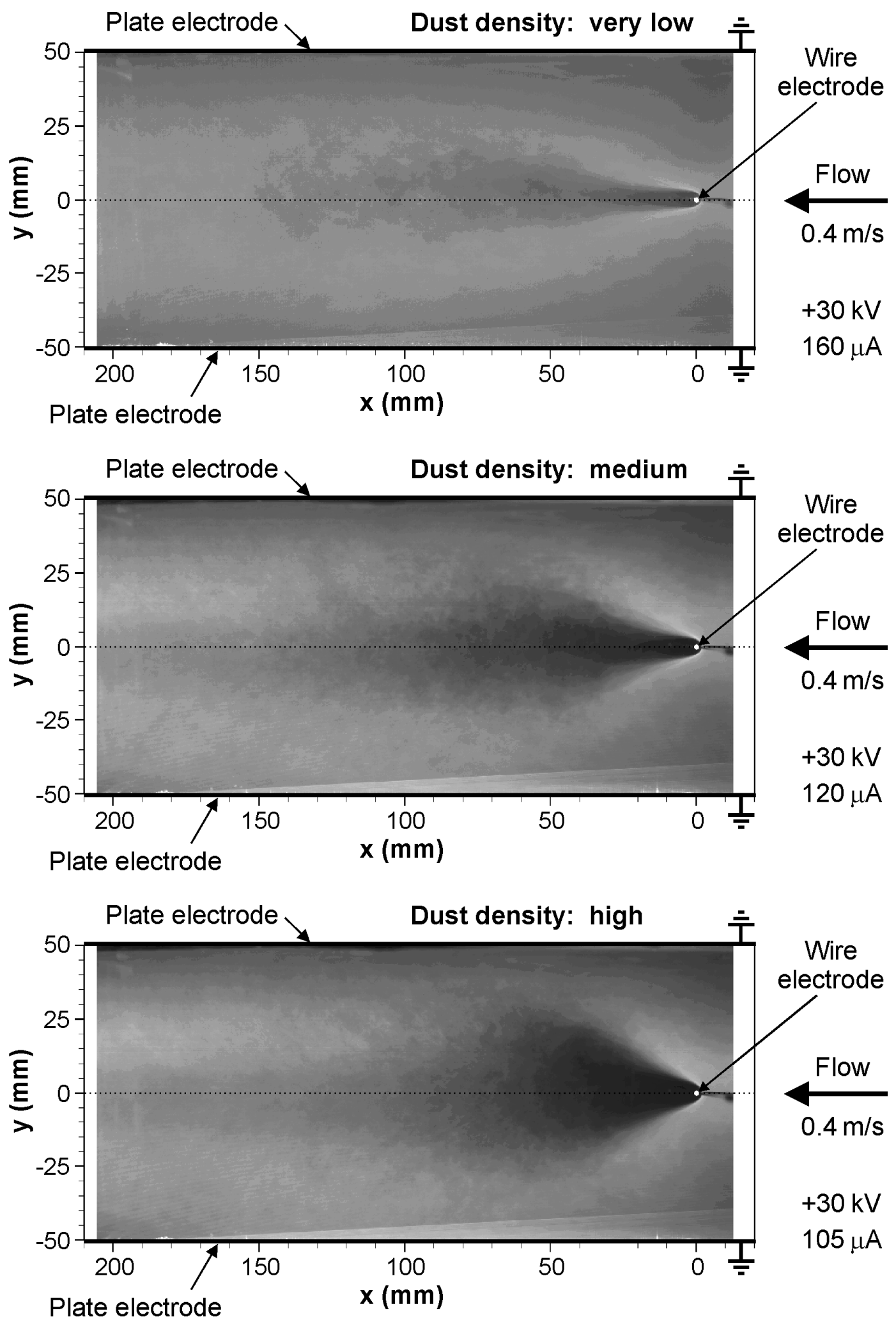

Fig. 9 - Averaged images (over 150 instantaneous images) of the particle flow in the ESP (primary flow velocity : $0.4 \mathrm{~m} / \mathrm{s}$, applied voltage $:+30 \mathrm{kV}$ ) in the same conditions for dust density and greyscale as in Fig. 8. 

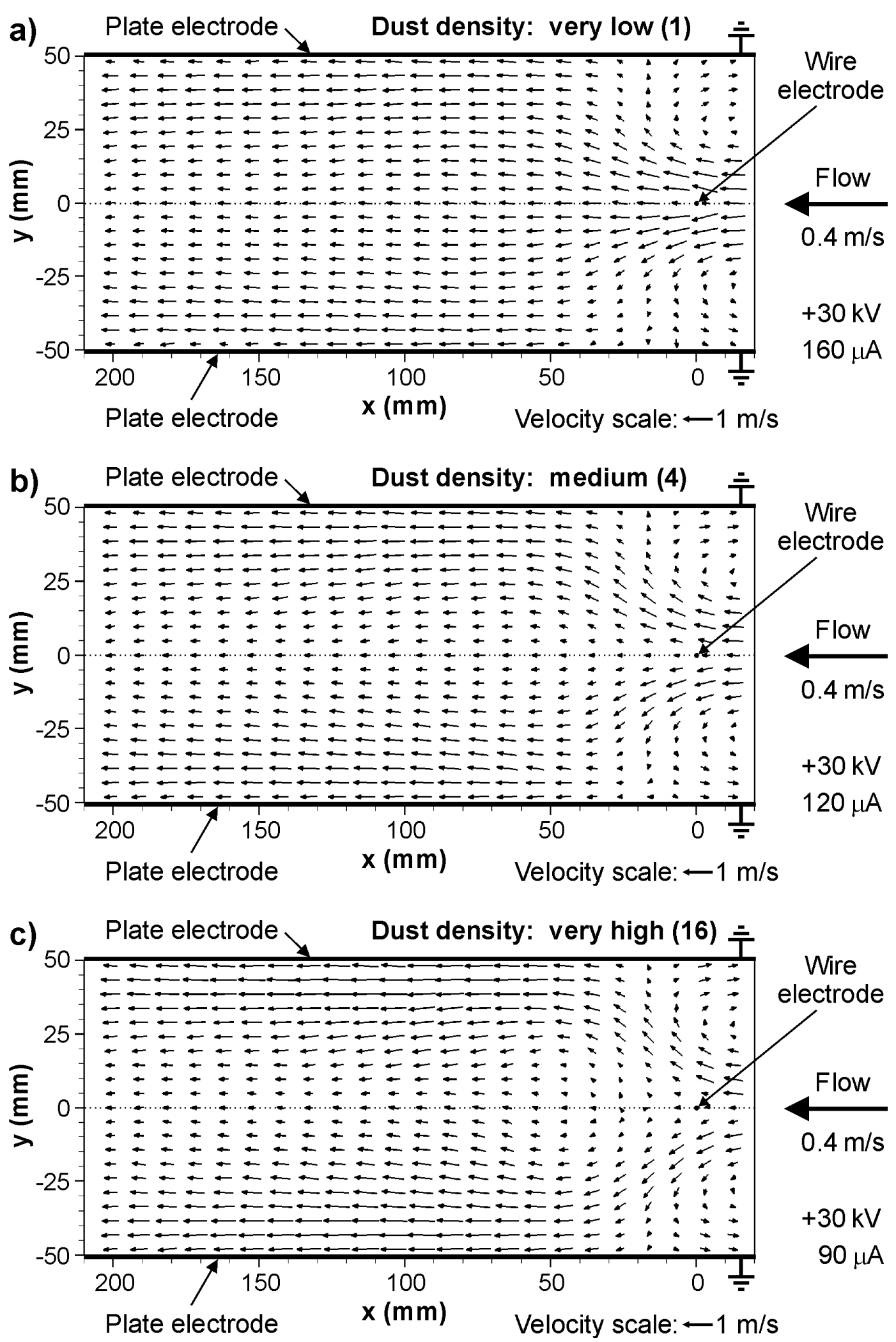

Fig. 10 - Averaged particle flow velocity fields in the ESP for different dust densities. The primary mean gas velocity was $0.4 \mathrm{~m} / \mathrm{s}$. The applied voltage was $+30 \mathrm{kV}$. Total discharge current: a) $160 \mu \mathrm{A}$, b) $120 \mu \mathrm{A}$, c) $90 \mu \mathrm{A}$. 

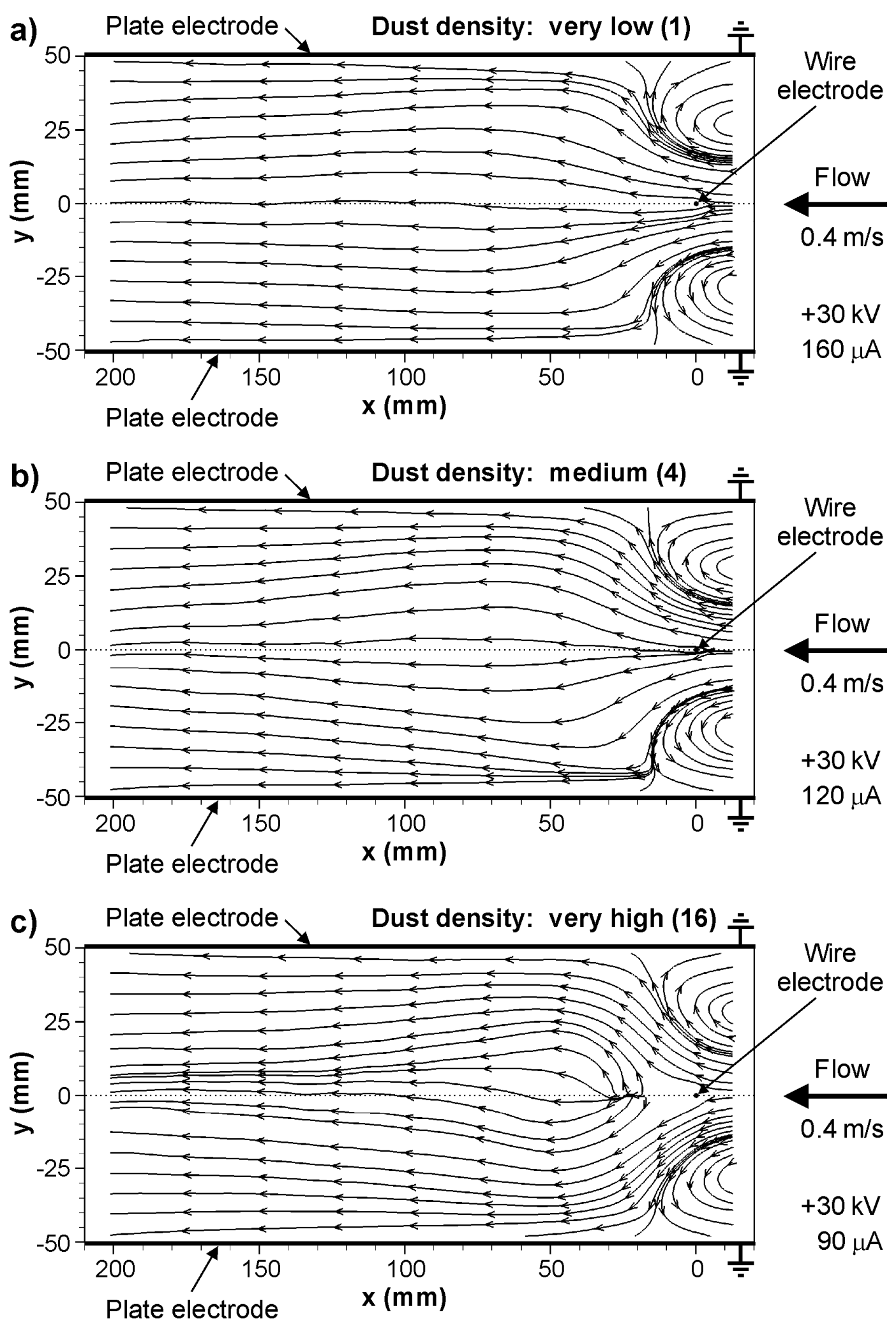

Fig. 11 - Particle flow streamlines in the ESP for different dust densities. The primary flow velocity was $0.4 \mathrm{~m} / \mathrm{s}$. The applied voltage was $+30 \mathrm{kV}$. Total discharge current:
a) $160 \mu \mathrm{A}$,
b) $120 \mu \mathrm{A}$,
c) $90 \mu \mathrm{A}$. 

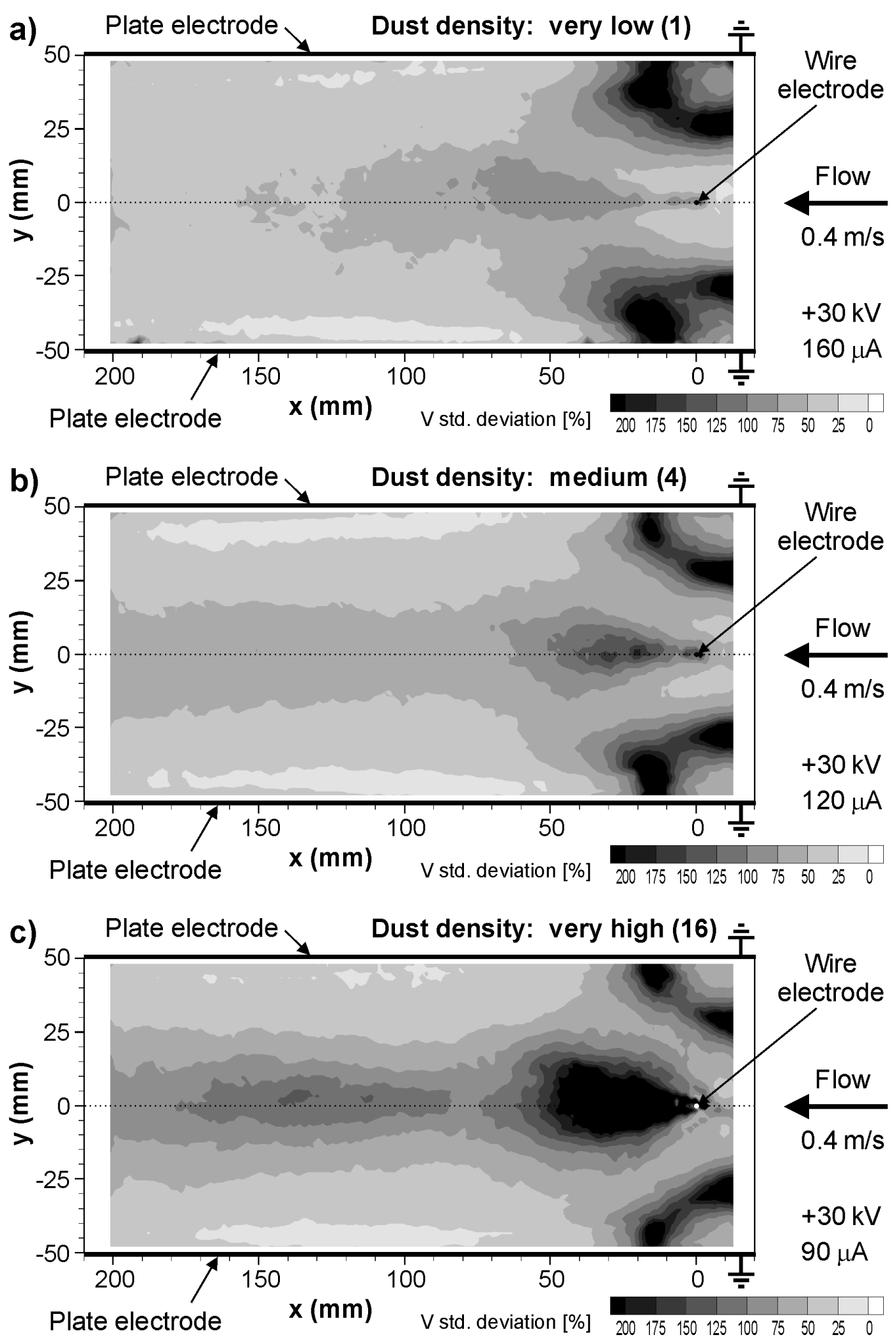

Fig. 12 - Particle flow velocity standard deviation in the ESP for different dust densities. The primary flow velocity was $0.4 \mathrm{~m} / \mathrm{s}$. The applied voltage was $+30 \mathrm{kV}$. Total discharge current: a) $160 \mu \mathrm{A}$, b) $120 \mu \mathrm{A}$, c) $90 \mu \mathrm{A}$. 